\title{
REMLABNET IV - LTI Federated Remote Laboratory Management System with Embedded Multiparameter Simulations
}

\author{
https://doi.org/10.3991/ijoe.v13i10.7802 \\ Franz Schauer( $\left.{ }^{\varpi}\right)$,Miroslava Ozvoldova, Michal Gerza, Michal Krbecek, Pavel Beno, \\ Tomas Komenda, Sayan Das, Mbuotidem Ime Archibong \\ Tomas Bata University in Zlin, Zlin, Czech Republic \\ fschauer@fai.utb.cz
}

\begin{abstract}
The recent progress in REMLABNET (www.remlabnet.eu ), namely its LTI Federation possibilities with other systems and multiparameter simulations embedded, is presented together with the main recent features of REMLABNET (as a Remote Laboratory Management System). At present the available real remote experiments have been provided for Go-Lab EU FP 7 project. The system with about 20 remote experiments has been with success running since 2013 and has been since constantly improved thanks to the project of the Swiss National Science Foundation (SNSF) - "SCOPES".
\end{abstract}

Keywords - Internet School Experimental System, Remote Laboratory Management System, REMLABNET, remote experiments, LTI connectivity, embedded simulations

\section{Context - Introduction}

Federated remote laboratory management system, REMLABNET IV (version 2017), for the integrating and management of remote experiments, is presented. Its building was initiated both from the extensive use and expertise in Internet School Experimental System (ISES) and the lack of a similar system for secondary schools in Europe. The history and reasons for RLMS system REMLABNET are to be found in the original paper [1].

The system uses new features added to REMLABNET in 2016, namely:

- Learning Tools Interoperability (LTI) federation to any arbitrary system as, e.g. LMS system MOODLE (inclusive Go-Lab), into one cooperating system sharing remote experiments,

- New Measureserver (2016) with the ability to embed multi parameter simulations to model remote experiment data.

The paper is a free continuation of the papers on Remote Laboratory Management System (RLMS) REMLABNET II published in REV 2015 in Bangkok [2] designed for integrating and management of remote experiments (REs) for secondary schools 
and the university level with the special focus on its role in research- based teaching and REMLABNET III published in REV 2016 in Madrid, where we dealt with the problem of Go-Lab federation [3].

\section{Purpose of REMLABNET}

General purpose of the Remote Laboratory Management System (RLMS) is to provide the arbitrary client with REs contained in the system of the Trnava University (http://www.remlabnet.eu/list.pdf) with REs of the Consortium [4] from Trnava, Zlin and Prague. The experiments are provided with double level diagnostics and in progress is embedding of multiparameter simulations, accompanying the REs.

\section{Functionality and approach of REMABNET}

\subsection{Federation and LTI of RLMS}

In 2015 we succeeded in two basic steps, in cooperation with the technical committee of the EU FP 7 Go-Lab we devised the interconnection of both Go-Lab (D. Gillet) and REMLABNET RLMS (F. Schauer) systems creating two federated remote laboratory management systems for the first time. The second step was accomplished when Consortium [4] REs were interconnected to the Go-Lab for sharing, which process continues till now (see Figure 1).Learning Tools Interoperability (LTI) is the product developed by IMS Global Learning Consortium [http://www.imsglobal.org/activity/ learning-tools-interoperability ]. The principal concept of LTI is to establish a standard way of integrating rich learning applications (often remotely hosted and provided through third-party services) with platforms like learning management systems, portals, learning object repositories, or other educational environments. This approach was adopted by many big systems like a Moodle, Google course builder and many more. In LTI these LMS, or platforms, are called Tool Consumers and the learning applications are called Tools (delivered by Tool Providers) (See Figure 1). Advantage is a security which is ensured between provider and costumers. When end user is logged into costumer system there is no need to log into provider system. We adopted LTI to Remlabnet and create new API for it. At this moment there is a full integration of LTI which provides end users' and costumer systems' metadata. This allows us to introduce new functionality like a storing of measured data, single user statistic and direct feedback for users of the costumer system.

\subsection{Multiparameter simulations embedded in remote experiments of RLMS}

Students have sometimes problems to understand complex phenomena behind the ISES remote experiments and the physics laws governing the experiment. To provide insight into physics of the phenomenon in question, a multiparameter simulation, working with analytical formulations of the respective physics law might be of a great 


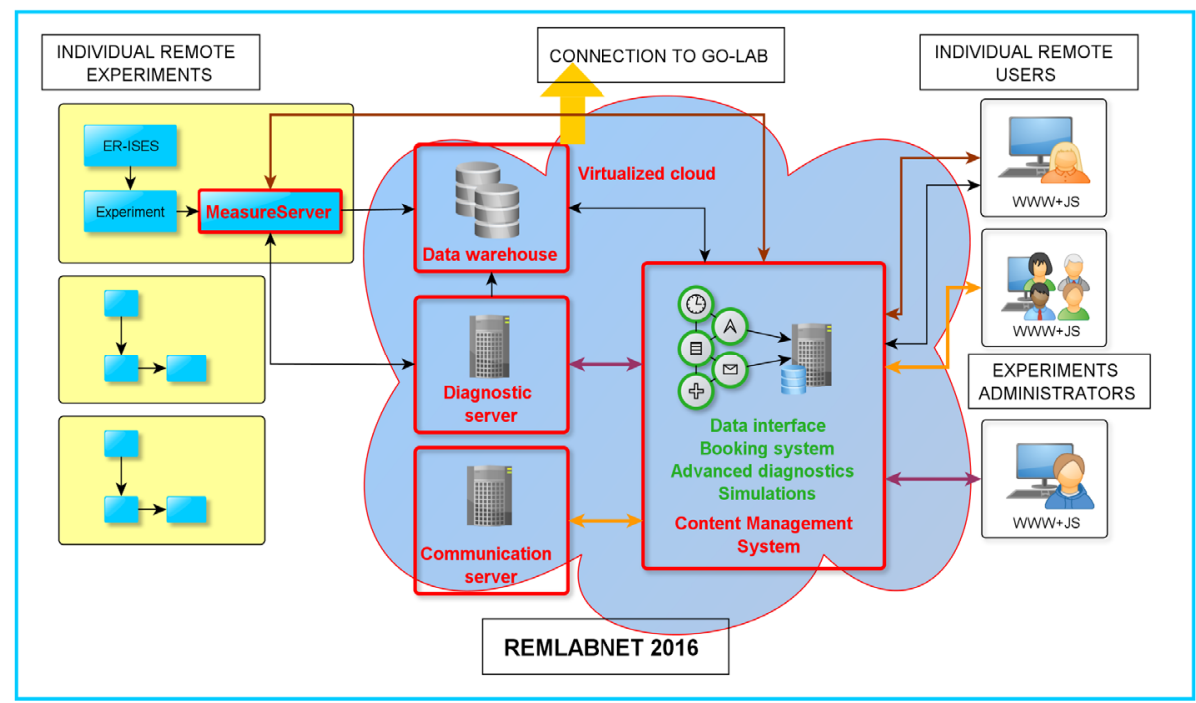

Fig. 1. Schematic representation of federated remote laboratory management system (RLMS) - REMLABNET (www.remlabnet.eu), built by the consortium of Tomas Bata University in Zlin, Charles University in Prague and Trnava University in Trnava and interconnected to the Go-Lab portal (www.go-labz.eu) the connectivity to the LTI is provid-

ed

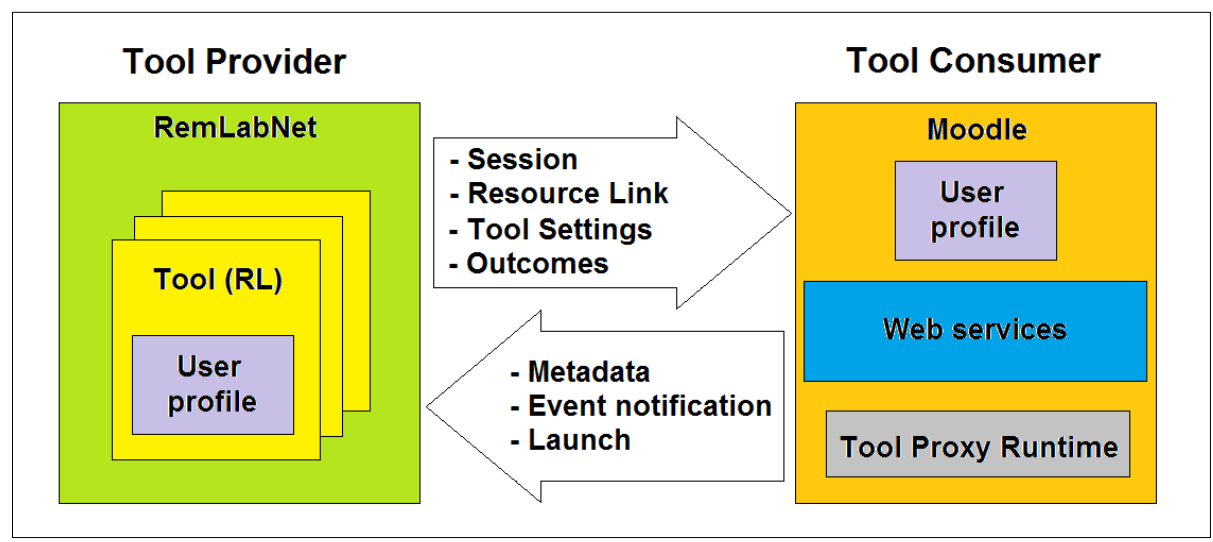

Fig. 2. The general scheme for Remlabnet with the interface Learning Tools Interoperability (LTI)

help. For this purpose, a new module has been designed and implemented into remote experiment software, enabling simulations embedding into remote experiments outputs. This module is a part of the Measureserver (MS) called phenomena simulation module (PSM). The MS is a program core unit deployed for the data gathering, processing and distribution. It resides in underlying structure of the ISES remote experiment (RE) between the physical apparatus and the REMLABNET platform. The PSM 
runs concurrently with the ISES RE as a physical model. Both models are entirely synchronized during the experimentation, the PSM enables variable model coefficients. The core of the PSM are Runge-kutta and Modified Euler solvers for ordinary differential equations of the first and second order.

As an example, the RLC circuit with the artificially introduced damping was chosen (Figure 3). The circuit consists of a real capacitor and real inductor and two variable damping resistors, one in series and one in parallel with the circuit. In Figure 3 are the time domain responses of the RLC instantaneous current $i(t)$ to a unit step voltage $U$ perturbation both in real experiment (red) and simulation (blue). The PSM starts the solver to calculate the solution with adjusted parameters at an identical time with the remote experiment. Two situations are depicted, one with not fitted simulated data with respect to the measured data (left) and the $100 \%$ fit (right).


Fig. 3. The time domain response of RLC circuit to step Remote experiment (red) with Embedded multiparameter simulation (blue). Left: Remote experiment data not fitted by simulation data, Right: Remote experiment data $100 \%$ fitted by simulation data

\subsection{New arrangement of Remlabnet and ISES e-experiments of RLMS [1]}

JavaScript redefining. Due to the restrictions, imposed on Java applets in 2013, we have had to completely rewrite the controlling programs of all our remote experiments and to impose changes in experiment themselves. In searching for more suitable web communication language we choose JavaScript and in July 2015 all our remote experiments were ready to use again. The outcomes pros outweighed the efforts exerted, as we brought to the young generation remote laboratories accessible anywhere, anytime and by any communication means ranging from smartphones to notebooks. By this step we also brought the remote laboratories nearer to schools and practical education. In Figure 4 is the front page of a new Remlabnet (see www.remlabnet.eu) and the offer of available remote experiments, and in Figure 5 an example of rewritten web page of the experiment "Simple pendulum" with Java applets (up) and JavaScript. 


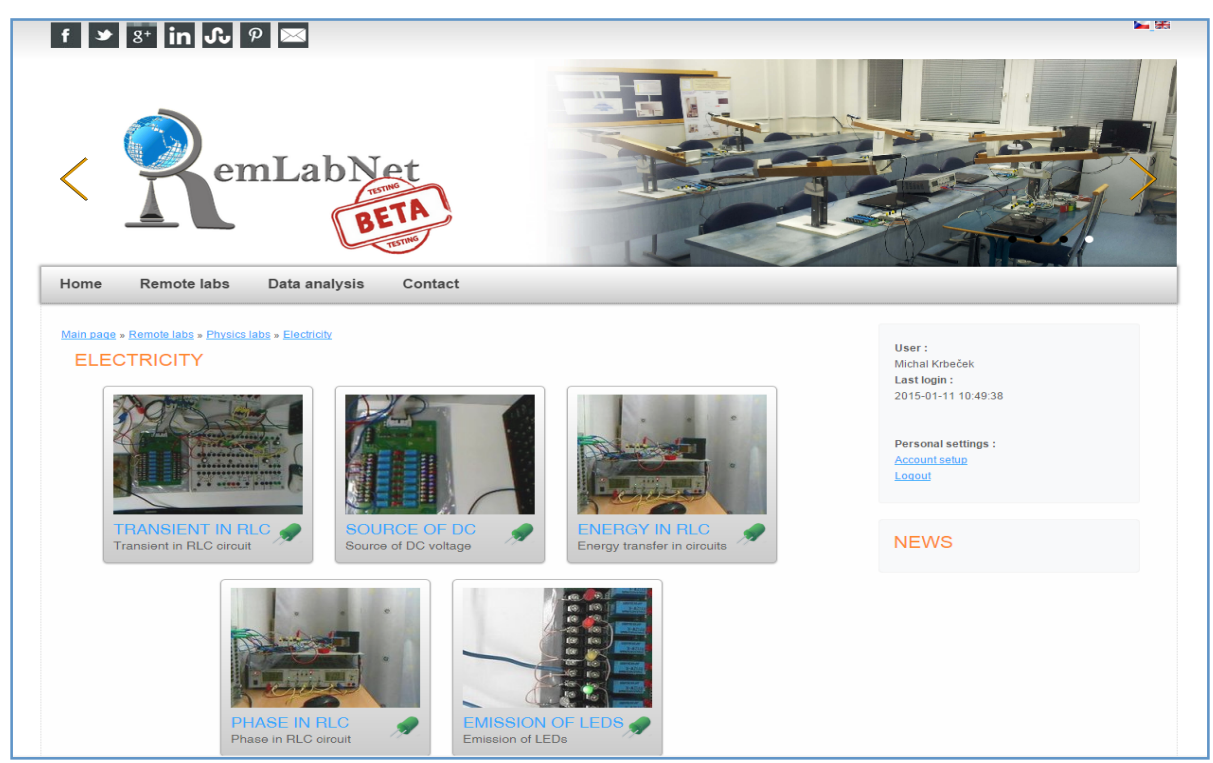

Fig. 4. Content management system - Remlabnet: with offer of remote experiments ( see http://www.remlabnet.eu )

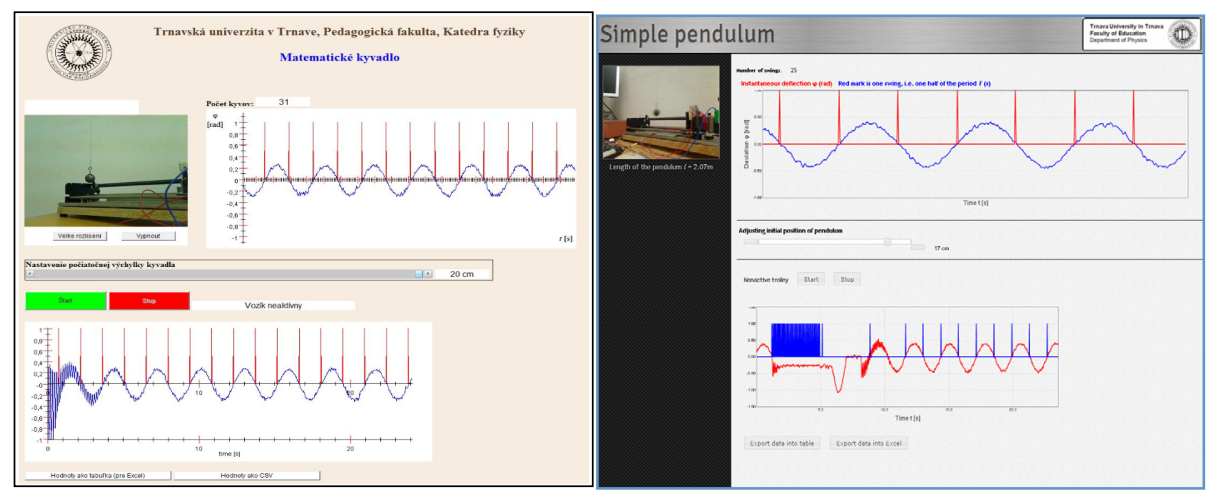

Fig. 5. www front page of the remote experiment "simple pendulum"; version by java applets 2013 (left) and JavaScript 2015 (right)

\subsection{ISES e-experiments with two level Diagnostics of RLMS [3]}

The crucial drawback of existing REs is the lack of feedback of their functionality. The clients are then disgusted and are repelled from the regular use of REs. In our current Measureserver@we accommodated two diagnostic systems, whose flow chart diagram is in Figure 6. The System I (Figure 7) signals by the "traffic lights" the available experiment ((a) green light), occupation of the experiment (temporary or permanent) ((b) orange light) and out of order experiment - not available for the service - (red light (c)). 


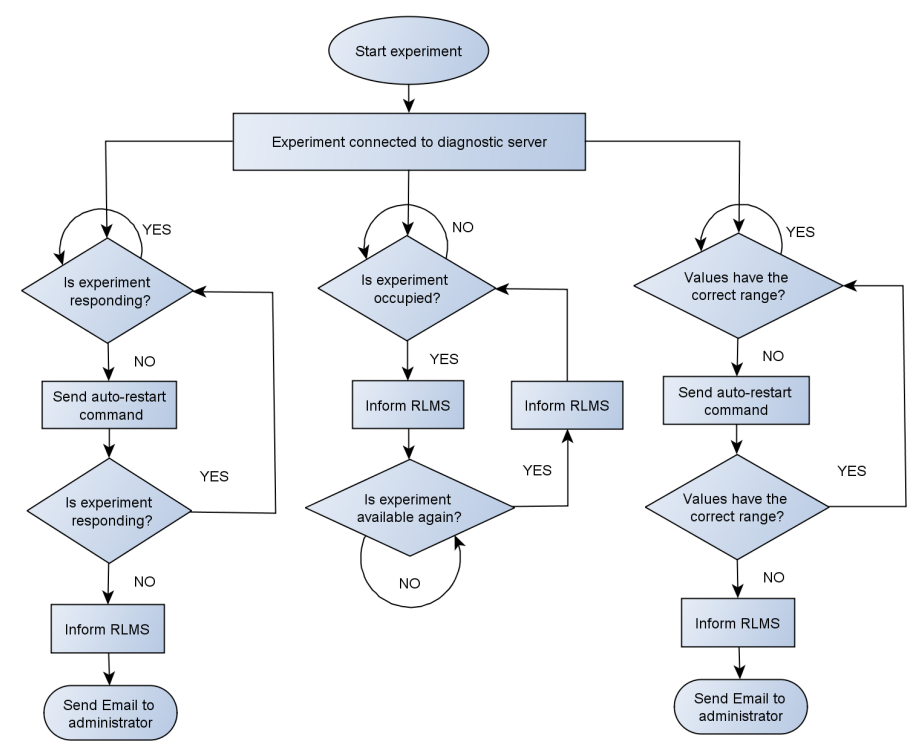

Fig. 6. Flow chart diagram of two diagnostic loops of REMLABNET remote experiments
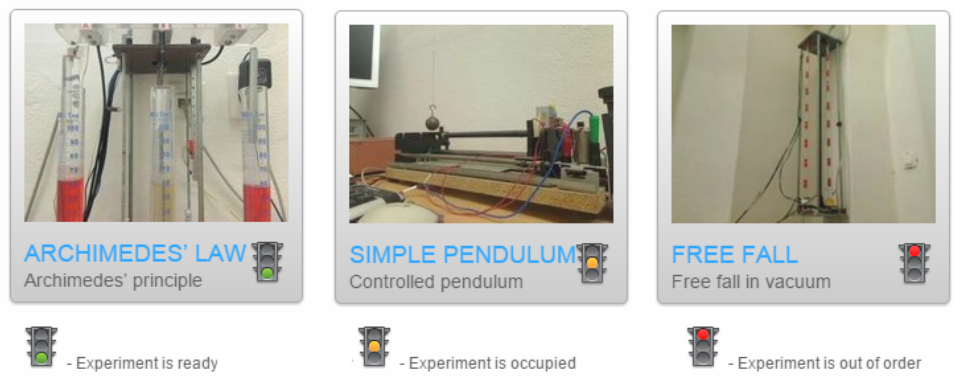

Fig. 7. Remote experiment Diagnostics I - the "traffic lights" signal availability of the experiment (green), occupation of the experiment (orange) and out of order experiment - not available (red).

System Diagnostics II is a more sophisticated diagnostic system, watches for the proper functioning of all the components of the RE, displaying the continuous reports in regular time intervals of the state of the physical hardware. By green color are denoted the correct set up and functions. In Figure 8a,8b are indicated two typical fault events. If the range or the sensitivity of any modules changes (see the event A in Figure 8b, yellow color), or if any module is disconnected (see the event B in Figure 8b, yellow color), the corresponding report occurs and e-m message is sent to the experiment owner as request. At the beginning of the remote experiment programming the ISES components and modules of the experiment are collected in the reference list with their sensitivity or ranges (see Figure 8a). 


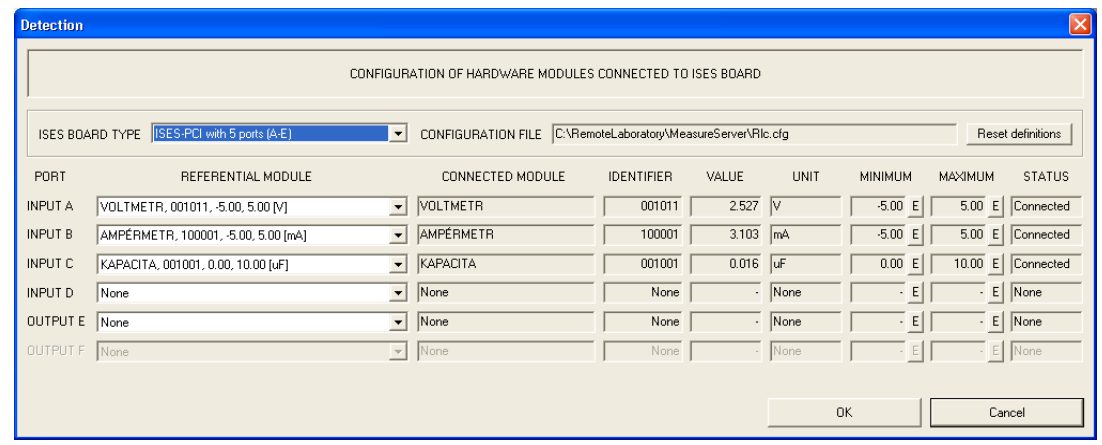

Fig. 8a Reference list providing with details of ISES physical HW modules

\begin{tabular}{|c|}
\hline$[10: 29: 00]$ [Measureserver is running] \\
{$[10: 29: 30]$ [Measureserver is running] } \\
{$[10: 29: 50]$ [Measureserver is running] } \\
\hline (A) \\
{$\left[\begin{array}{c}\text { [10:30:30] [PORT:B, NAME:AMPERMETR, ID:000001, UNIT:A, MIN:-0.50, MAX:0.50, STATUS:Connected with } \\
\text { wrong reference] }\end{array}\right.$} \\
\hline$[10: 31: 00]$ [Measureserver is running] \\
{$[10: 31: 10]$ [Measureserver is running] } \\
{$[10: 31: 20]$ [Measureserver is running] } \\
\hline (B) \\
\hline [10:32:00] [PORT:B, NAME:AMPERMETR, ID:000001, UNIT:A, MIN:-0.50, MAX:0.50, STATUS:Diconnected] \\
\hline$[10: 35: 00]$ [Measureserver is running] \\
{$[10: 35: 20]$ [Measureserver is running] } \\
{$[10: 35: 30]$ [Measureserver is running] } \\
\hline
\end{tabular}

Fig. 8b Remote experiments Diagnostics II record; correctly running experiment (green) and two faults (yellow) the setup of the remote experiment changed (range of A-meter) (case A), one module A-meter disconnected (case B)

\begin{tabular}{|c|}
\hline$[10: 29: 00]$ [Measureserver is running] \\
{$[10: 29: 30]$ [Measureserver is running] } \\
{$[10: 29: 50]$ [Measureserver is running] } \\
\hline (A) \\
\hline 10:30:30] [PORT:B, NAME:AMPERMETR, ID:000001, UNIT:A, MIN:-0.50, MAX:0.50, STATUS:Connected with \\
wrong reference] \\
\hline$[10: 31: 00]$ [Measureserver is running] \\
{$[10: 31: 10]$ [Measureserver is running] } \\
{$[10: 31: 20]$ [Measureserver is running] } \\
\hline (B) \\
\hline [10:32:00] [PORT:B, NAME:AMPERMETR, ID:000001, UNIT:A, MIN:-0.50, MAX:0.50, STATUS:Diconnected] \\
\hline$[10: 35: 00]$ [Measureserver is running] \\
{$[10: 35: 20]$ [Measureserver is running] } \\
{$[10: 35: 30]$ [Measureserver is running] }
\end{tabular}




\subsection{Virtualized cloud and services of RLMS [2]}

Virtualized Cloud - (Schematic functioning of virtualization is in Figure 9) To provide optimal access to all the experiments and economical exploitation of the RLMS with its all functionalities and benefits, we intend to use the virtualized cloud computing.

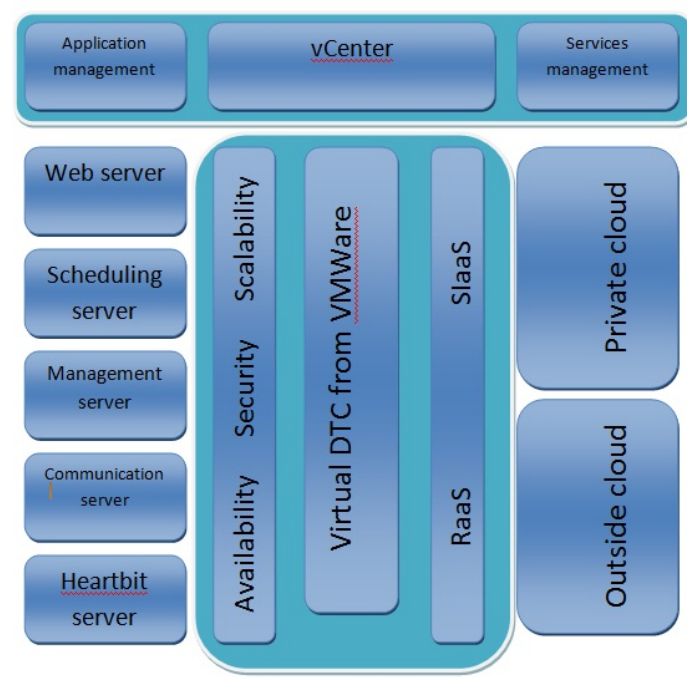

Fig. 9. Schematic functioning of cloud virtualization

Our idea is make two or more datacenters (DTC) with relevant data and clients connected to the nearest DTC with the lowest traffic and utilization. Because of a large number of inexperienced users may access RLMS, we need to create as secure a network environment as possible. Specific concern is the RE computer system, the software from instrument vendor, and the security of data collected.

For our work are dedicated few servers in two datacenters. Vendor of this servers are Oracle (SunFire) and Cisco (UCS). All of servers are with ESXi operating system from VMWare and with one vCenter for management of them. We can use for the purpose following features of the system [20]. Operating systems for each VMs are used by purpose (for example MS Windows desktop or server editions, Linux SLES for VMWare or Ubuntu etc.)

Security of DTCs is built on several levels. First security level is based on IP (Internet Protocol) with allowed or denied IP addresses and ports. Second security concern will deal with the unauthorized access to the instrumentation and computer desktop, is user level with encrypted usernames and passwords used SSL to transmit between DTCs. Third level is IDS (intrusion detection system) and IPS (intrusion prevention system) to monitoring possible incidents. Fourth and last is SIEM (security information and event management) and Checkpoint application firewall for monitoring on the application level. 


\section{REMLABNET- feature and outcomes}

In near future we will be dealing with following topics: interface recognition and connection - One of the envisaged and highly required qualities of RLMS REMLABNET is the necessity of recognizing of variety of common interfaces, the elaboratories may possess.

On applying to the RLMS, the system responds by the connecting of the corresponding transformation driver, transforming a rig's data output to XML format. On the other hand, the system behaves in the bidirectional way, transforming the data sent by the client to the format of the rig. In such a way the system will by quite universal, removing all the communication barriers.

- Connectivity of REMLABNET to MOODLE by LTI - all running courses of Physics in MOODLE will be provided with direct connectivity to REMLABNET, so making easy a direct approach to all RE and accompanying material during teaching without boring delays.

- Registration of RE in RLMS - As a communication protocol for the data processing with the server JSON (Java Script Object Notation) or XML (Extensible Markup Language) will be used. Once the connection is established, the system will send a crafted web page to a web server and ensure experiment inclusion in the database with all necessary information that the user enters during the experiment setup. This information should allow the inclusion of experiment in the appropriate category, describing its physical background and its functioning. Descriptions will not be restricted to mere textual information, but will allow inserting of images and videos in order to achieve the deepest possible problem understanding. By this step the experiment is included in the database and available on the web portal REMLABNET.

- Virtual Classroom - This service will allow the integration of the rig and entry of the users into a virtual classroom. The virtual class setup will also enable (student / teacher) roles allocation Virtual classroom will provide special features for testing and evaluation of knowledge of the students as well. Communication within the virtual classroom will be by the video conferencing or text-only chat. For this purpose communication protocols like VOIP (voice over Internet Protocol) and RTSP (Real Time Streaming Protocol) will be used.

- Communication board - This service will be a simple communication window that will serve to put questions to administrator or the insertion of proposals for improvements and feedback by email. This feature will be fully automated. Communication with the administrator will be displayed on the whiteboard window.

- Entrance test - This function will restrict the access to the experiment only to those, who passed the test entered by the administrator. It will test the user's knowledge and prevent misuses of the experiment. The results of these tests will be stored and may be used for the statistical purposes.

- Transformation of rig's data to XML. The main purpose of this transforming interface is to gather experiment configuration, current controlling values and measured data results in order to maintain particular experiment setup and repro- 
duce it off-line. Every rig is planned to be equipped with a separated storage space which should include the date, time, logged user and description, identifying the experiment and the measured data gathered from the experiment physical hardware (apparatus). This interface, giving data in standardized XML, will be used for a simulation process.

- Database storage and its evaluation using artificial intelligence - The system will provide storage of all information concerning experiments and enable their displaying in the form of a catalogue. In database there will be stored measured experimental data of registered users for later use.

\section{Acknowledgments}

The support of the project of the Swiss National Science Foundation (SNSF) "SCOPES" No IZ74Z0_160454 is highly appreciated. Support of the Internal Agency Grant of the Tomas Bata University No. IGA/FAI/2016 for all cooperating PhD students is acknowledged.

\section{References}

[1] F. Schauer, M. Krbecek, P.Beno, M. Gerza, L. Palka and P. Spilakova: REMLABNET open remote laboratory management system for e-experiments, in Proc. of REV 2014, Porto, Portugal. pp 268-273. https://doi.org/10.1109/REV.2014.6784273

[2] F. Schauer, M. Krbecek, P.Beno1, M. Gerza, L. Palka and P. Spilakova: REMLABNET II - open remote laboratory management system for university and secondary schools research based teaching, in Proc. of REV 2015, Bangkok, Thailend. pp 249-242

[3] Franz Schauer, Michal Krbecek, Pavel Beno, Michal Gerza, Lukas Palka, Petra Spilaková and Lukas Tkac: REMLABNET III - federated remote laboratory management system for university and secondary schools, in Proc. 13th International Conference on Remote Engineering and Virtual Instrumentation, REV 2016, Madrid; 25 -27 February 2016, pp 232235.

[4] The Consortium of Remlabnet (www. remlabnet .eu) is formed by three laboratories in Faculty of Education, Trnava University in Trnava, next, Faculty of Informatics, Tomas Bata University in Zlin and Faculty of Mathematics and Physics, Charles University in Prague.

\section{Authors}

Franz Schauer received a M.S. degree in Electronics from the Brno University of Technology in 1963 and his Ph.D. degree in Solid State Physics from Prague University of Technology in 1978. In 1982 he was appointed Associate Professor and in 1988 Professor in Condensed Matter Physics at the Technical Academy in Brno, Czech Republic. In 1993-2002 he was with the Faculty of Chemistry, Brno University of Technology and since then he was with the Polymer Centre of the Faculty of Technology. At present he is with the Tomas Bata University in Zlín, Faculty of Applied 
Informatics, Czech Republic. His main activities are molecular organic electronics, electronic structure spectroscopy by electrochemical and charge injection methods and computer assisted experiments. He is the author of about 300 papers in both Solid state physics, Education practice and Remote laboratories with about 350 SCI citations.

Miroslava Ozvoldova received her M.S. degrees in Physics from Comenius University in Bratislava, Slovakia, in 1973, and in 1981 a Ph.D. in Physics-Mathematics Science. In 1992 she was appointed Associate Professor, and in 2002 Extraord. Professor at the Faculty of Materials Science and Technology in Trnava, Slovak University of Technology in Bratislava. Since 2008 she is with the Faculty of Informatics, Tomas Bata University in Zlin. Her main activities are optical properties of chalcogenide and heavy metal optonic glasses and e-learning in Physics teaching.

Michal Gerza, Michal Krbecek, Pavel Beňo, Tomas Komenda, Sayan Das, Mbuotidem Ime Archibong are $\mathrm{PhD}$ students with the Tomas Bata University in Zlín, Faculty of Applied Informatics, Nad Stráněmi 4511, Zlín, CZ- 760 05, Czech Republic.

Article submitted 07 September 2017. Published as resubmitted by the authors 13 October 2017. 